\title{
Clinical Interventions to Promote Breastfeeding by Latinas: A Meta-analysis
}

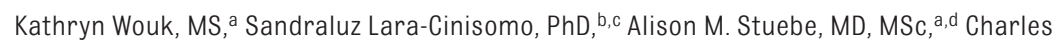

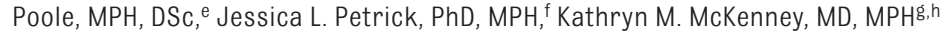

CONTEXT: Breastfeeding duration and exclusivity among Latinas fall below recommended levels, indicating a need for targeted interventions. The effectiveness of clinical breastfeeding interventions for Latinas remains unclear.

OBJECTIVE: To systematically review the documented effectiveness of clinical breastfeeding interventions on any and exclusive breastfeeding among Latinas.

DATA SOURCES: English-language publications in Medline, CINAHL, and Embase were searched through May 28, 2015.

StUdY SElEction: Fourteen prospective, controlled studies describing 17 interventions met inclusion criteria.

DATA EXTRACTION: Extracted study characteristics include study design, population characteristics, intervention components, timing and intensity of delivery, provider type, control procedures, and outcome measures.

RESULTS: Random-effects meta-analyses estimated risk differences (RDs) between breastfeeding mothers in intervention and control arms of each study and 95\% prediction intervals (PIs) within which 95\% of intervals cover the true value estimated by a future study. Interventions increased any breastfeeding at 1 to 3 and 4 to 6 months (RD 0.04 [95\% PI -0.15 to 0.23 ] and 0.08 [ -0.08 to 0.25$]$, respectively) and exclusive breastfeeding at 1 to 3 and 4 to 6 months ( 0.04 [ -0.09 to 0.18 ] and 0.01 [ -0.01 to 0.02$])$. Funnel plot asymmetry suggested publication bias for initiation and 1- to 3-month any breastfeeding. Estimates were slightly larger among interventions with prenatal and postpartum components, 3 to 6 patient contacts, and delivery by an International Board Certified Lactation Consultant or lay provider.

LIMITATIONS: The published evidence for Latinas is limited, and studies have varying methodologic rigor.

conclusions: Breastfeeding interventions targeting Latinas increased any and exclusive breastfeeding compared with usual care.

\footnotetext{
${ }^{a}$ Carolina Global Breastfeeding Institute, Department of Maternal and Child Health, and ${ }^{e}$ Department of Epidemiology, Gillings School of Global Public Health, University of North Carolina at Chapel Hill, Chapel Hill, North Carolina; ${ }^{b}$ Department of Psychiatry, School of Medicine, University of North Carolina at Chapel Hill, Chapel Hill, North Carolina; ${ }^{d}$ Department of Obstetrics and Gynecology, School of Medicine, University of North Carolina at Chapel Hill, Chapel Hill, North Carolina; ${ }^{\circ}$ Department of Kinesiology and Community Health, College of Applied Health Sciences, University of Illinois Urbana-Champaign, Champaign, Illinois; ${ }^{f}$ Division of Cancer Epidemiology and Genetics, National Cancer Institute, National Institutes of Health, Rockville, Maryland ${ }^{g}$ School of Medicine, University of North Carolina at Chapel Hill, Chapel Hill, North Carolina h Department of Obstetrics and Gynecology, Feinberg School of Medicine, Northwestern University, Chicago, Illinois
}

To cite: Wouk K, Lara-Cinisomo S, Stuebe AM, et al. Clinical Interventions to Promote Breastfeeding by Latinas: A Meta-analysis. Pediatrics. 2016;137(1):e20152423 
Breastfeeding is associated with a number of well-established health benefits for both mothers and infants. ${ }^{1}$ The American Academy of Pediatrics, American College of Obstetricians and Gynecologists, and American Academy of Family Physicians recommend exclusive breastfeeding for 6 months, with continued breastfeeding alongside complementary foods for 1 year or longer. ${ }^{2-4}$ Models suggest that current suboptimal breastfeeding in the United States is associated with $>900$ excess child deaths and $>4000$ potentially preventable maternal deaths annually. 5,6 Healthy People 2020 has established national objectives to increase breastfeeding initiation, duration, and exclusivity. ${ }^{7}$ Whereas $82.4 \%$ of Latinas initiated breastfeeding in 2011, only $27.9 \%$ continued any breastfeeding at 12 months and $20.8 \%$ exclusively breastfed at 6 months. ${ }^{8}$ Latinas indicate a strong desire to breastfeed, surpassing the $80 \%$ of US mothers who initiate ${ }^{9}$; however, Latina breastfeeding duration and exclusivity are lower than national averages and $\sim 30 \%$ below Healthy People 2020 targets. These data highlight a need for interventions that support Latinas to achieve breastfeeding goals.

Latinas in the United States experience some barriers to breastfeeding more frequently than mothers of other ethnicities. Latinas are more likely than white women to stop breastfeeding because of latching difficulty, ${ }^{10}$ pain or fear of pain, ${ }^{10,11}$ perception of insufficient milk supply or infant preference for formula, ${ }^{10,12,13}$ and modesty or embarrassment. ${ }^{10}$ Latinas are more likely than both white and African American women to cite inconvenience or interference with desired lifestyle ${ }^{10,11}$ and belief that only poor women breastfeed ${ }^{11}$ as obstacles impeding breastfeeding. Latinas also experience a number of culturally unique barriers to breastfeeding, including family and partner pressures, norms regarding privacy, and cultural beliefs surrounding maternal diet and infant weight. ${ }^{14}$ Additionally, Latina women are 2 to 3 times more likely than non-Latinas to experience postpartum depression, ${ }^{15,16}$ which is associated with shorter breastfeeding duration and increased infant health concerns. ${ }^{17-19}$

Latina mothers have a lower prevalence of exclusive breastfeeding at 6 months compared with whites, Asians, and women who identify as $\geq 2$ races. ${ }^{9}$ Latina mothers are more likely than white or African American mothers to mix breastfeeding with formula supplementation, ${ }^{20-22}$ especially when family support is limited and free formula is distributed at hospital discharge. ${ }^{10,23}$ Mixed feeding becomes more prevalent with longer acculturation, ${ }^{10,23,24}$ and this practice is associated with both shorter breastfeeding duration and increased risk of childhood obesity. 5,20,22

The Centers for Disease Control and Prevention recommend interventions delivered by health care professionals as a key strategy to support breastfeeding mothers and increase breastfeeding rates. ${ }^{25}$ Previous systematic reviews of clinical breastfeeding interventions have found that breastfeeding education and support improve initiation and duration through 6 months ${ }^{26}$ and increase both short- and long-term breastfeeding in the general population. 27

However, although a recent review qualitatively evaluated interventions targeting minority women, ${ }^{28}$ no review has focused exclusively on Latina women. As Latinos become the largest minority group in the United States, accounting for more than half of total population growth, ${ }^{29}$ they are burdened by high rates of both uninsurance ${ }^{30}$ and illnesses for which breastfeeding reduces risks, including childhood asthma and asthma-related hospitalization, ${ }^{31-33}$ diabetes, ${ }^{34}$ and obesity. ${ }^{35}$ Thus it is essential to identify evidence-based clinical interventions to increase breastfeeding in this population.

This systematic review and metaanalysis has 2 main objectives: (1) to estimate the absolute effects of clinical breastfeeding interventions on any breastfeeding and exclusive breastfeeding at varying time points among Latinas and (2) to identify methodologic and etiologic factors that might modify these effects.

\section{METHODS}

\section{Search Strategy}

This systematic review and metaanalysis assesses both qualitative intervention characteristics and quantitative estimates of effect to systematically summarize the extant literature. In accordance with Preferred Reporting Items for Systematic Reviews and MetaAnalyses guidelines, ${ }^{36}$ we searched for English-language publications in Medline, CINAHL, and Embase through May 28, 2015, with no specified start date to ensure a comprehensive review of available evidence for this understudied population. We used the MeSH terms "breast feeding," "lactation," and "Hispanic Americans" and the key words "breastfeed," "counseling," "health education," "medical advice," "health practitioner," and "clinical intervention."

\section{Study Selection}

For inclusion, we required that articles be published in a peerreviewed journal (research abstracts were excluded), describe a clinical breastfeeding intervention for which women were recruited in a health care setting, include a control or comparison group, be conducted in the United States, report any or exclusive breastfeeding outcomes, and enroll a study sample $\geq 50 \%$ Latina. For this review, "Latina" 
refers to women of Cuban, Mexican, Puerto Rican, South or Central American, or other Spanish culture or origin. ${ }^{29}$ Interventions originating from a variety of health care settings, regardless of provider type and intervention location, were considered for inclusion. Comparison or control groups were those that represented usual standard of care in the facility from which the intervention originated.

Two investigators independently screened all titles, abstracts, and full-text publications based on these inclusion criteria. In addition, 1 of these 2 investigators reviewed reference lists of included publications and a related systematic review $^{26-28}$ to identify additional publications. Fig 1 illustrates our search and selection process.

\section{Data Extraction and Quality Assessment}

Two investigators extracted descriptive data from the Methods section of each publication, including study design, population characteristics, intervention components, timing and intensity of the intervention, provider delivering the intervention, control procedures, and outcome measures (Table 1). To assess methodologic quality, we examined randomization procedures, initial comparability of groups, attrition, allocation concealment, outcome measures, and whether intervention and control groups were clearly defined. We also assessed adherence to intent-totreat principles, handling of missing data, and inclusion of appropriate covariates. Considering all these factors, the same 2 investigators assigned a qualitative rating (good, fair, or poor) to each study based on criteria adapted from the US Preventive Services Task Force (Supplemental Table 4). ${ }^{37}$ Where ratings assigned by the 2 investigators were discordant, a
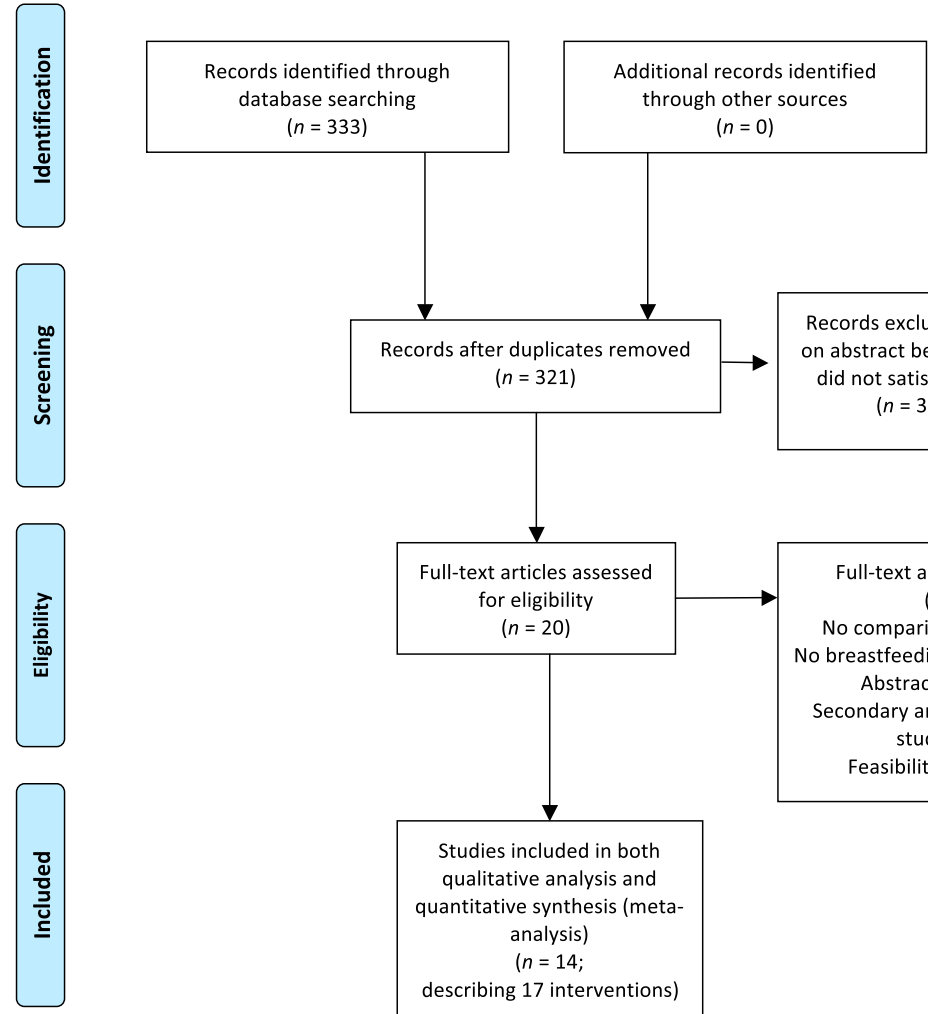

Records excluded based on abstract because they did not satisfy criteria $(n=301)$

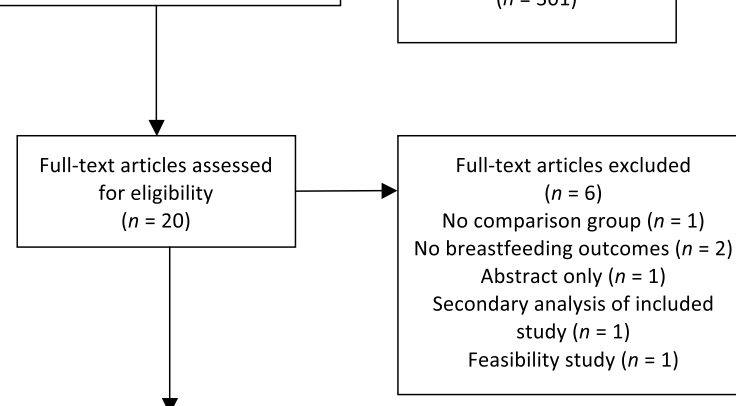

FIGURE 1

Search results and study selection procedures according to Preferred Reporting Items for Systematic Reviews and Meta-Analyses guidelines. ${ }^{36}$

final rating was reached through consensus.

\section{Breastfeeding Definitions}

Interventions may have differing effects on breastfeeding outcomes depending on the length of postnatal follow-up time. For consistency with outcome categorizations from previous meta-analyses, ${ }^{26,27}$ we defined breastfeeding prevalence at 3 different intervals: breastfeeding initiation at hospital discharge or within 2 weeks of delivery; shortterm breastfeeding at 1 to 3 months; and longer-term breastfeeding at 4 to 6 months. If a study reported outcomes at both 1 and 3 months or 4 and 6 months, we included in the meta-analysis the more commonly reported estimates from 3 and 6 months. Exclusive breastfeeding definitions were adopted from each study (Table 1).

\section{Data Synthesis and Analysis}

To estimate the absolute effect of breastfeeding interventions on any breastfeeding at each of the 3 time intervals and on exclusive breastfeeding at 1 to 3 months and 4 to 6 months, risk difference (RD) estimates, corresponding standard errors, and number needed to treat (NNT) were calculated. The RD is the difference in the proportion of breastfeeding mothers in the intervention $\operatorname{arm}\left(\mathrm{P}_{1}\right)$ and control $\operatorname{arm}\left(\mathrm{P}_{2}\right)$ of each study. Thus, a positive RD value suggests a beneficial intervention effect, and a negative $\mathrm{RD}$ value suggests that no benefit was gained from the intervention. The NNT is defined for each breastfeeding interval as $1 /$ $R D$, where positive values denote the estimated number of women who need to receive an intervention to result in 1 additional mother breastfeeding and negative values 


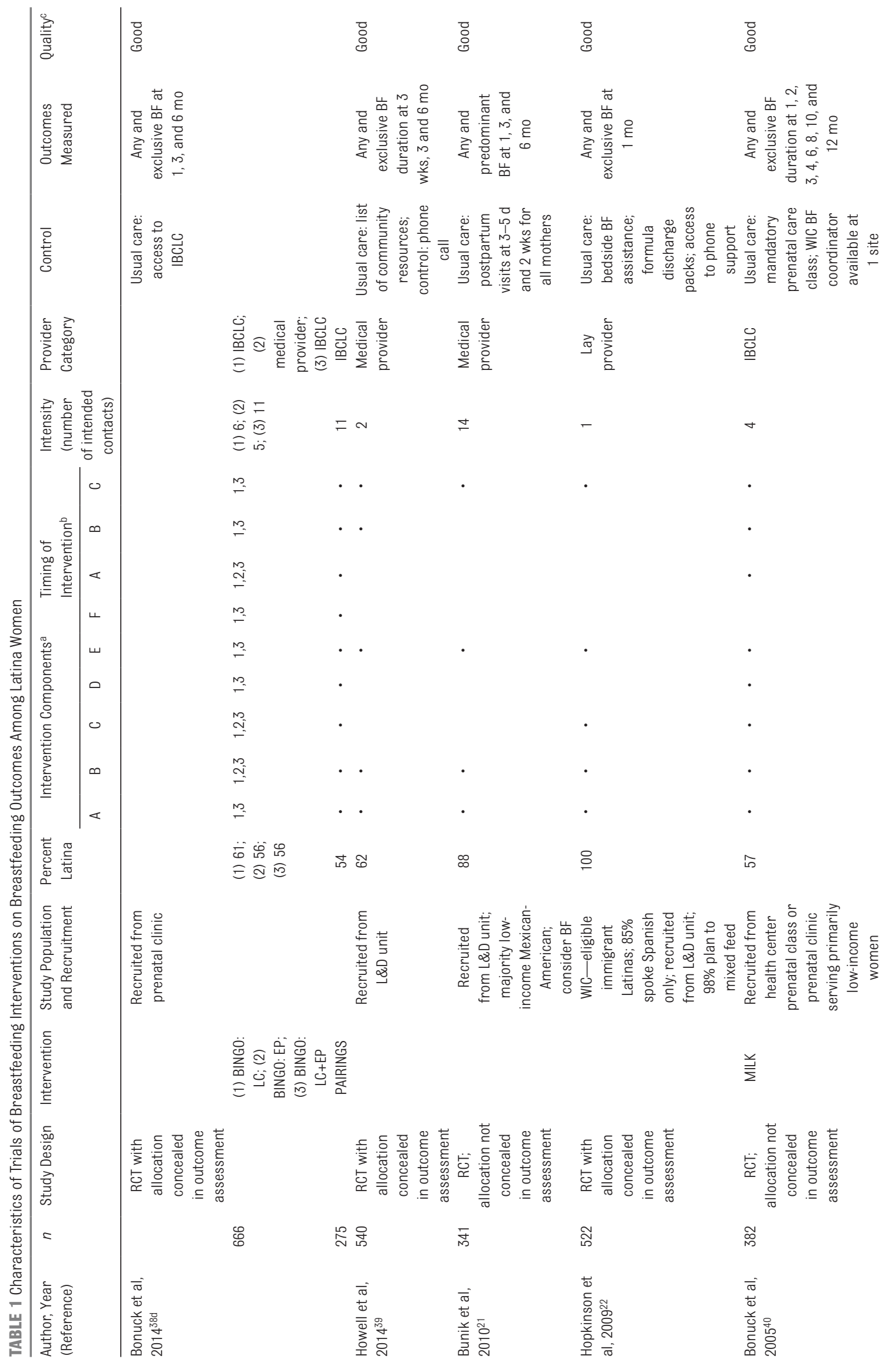




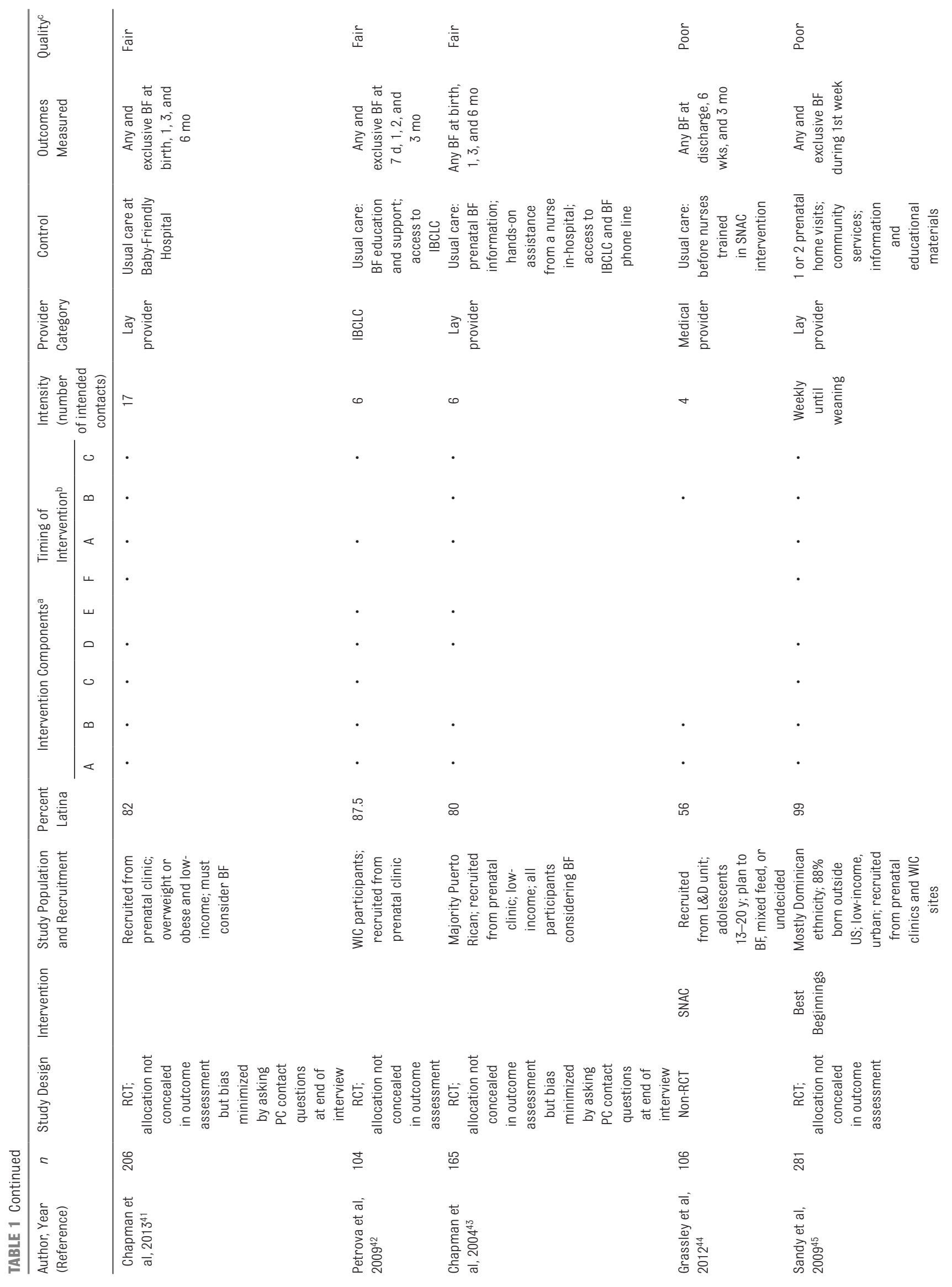




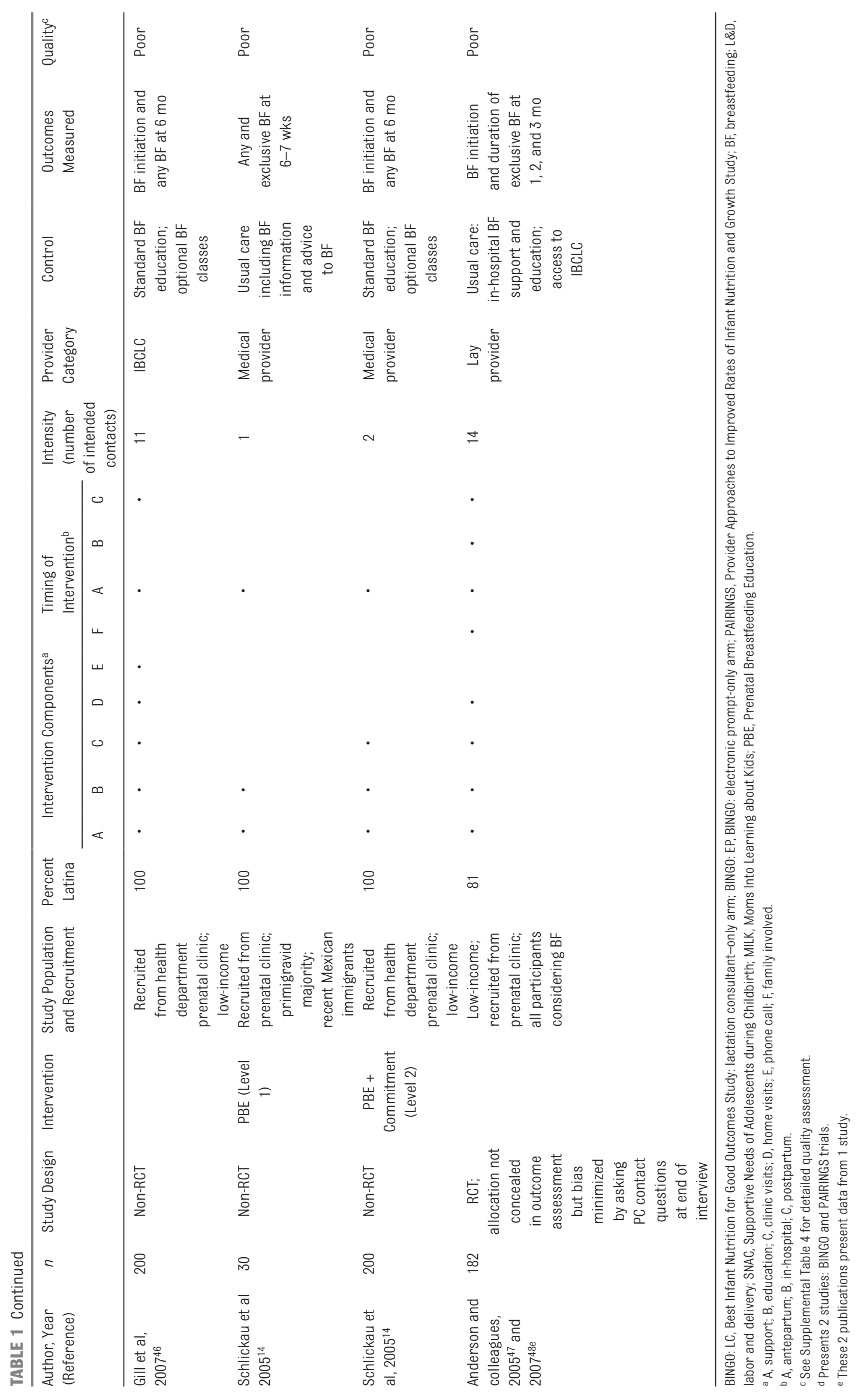


denote the number who need to receive an intervention to result in 1 fewer.

Two models were used for these analyses, a crude model and a univariable meta-regression model. The crude model was run to estimate the mean and variance of a random-effects distribution of RDs for any (Fig 2) and exclusive (Fig 3) breastfeeding stratified by time interval. The restricted maximum likelihood estimate of the amongpopulations variance $\left(\tau^{2}\right)$ and random-effects summarization were used to calculate summary estimates (Table 2). ${ }^{49} \tau^{2}$ is the variance of the presumptively normal distribution of true values among populations in which each population has its own true value (ie, its own true risk of breastfeeding). In each meta-analysis, we calculated a $95 \%$ confidence interval (CI) for the estimated mean $(\hat{\mu})$ of the presumptively normal distribution of population RDs:

$$
95 \% \mathrm{CI}=\hat{\mu} \pm 1.96 \widehat{S E},
$$

where $\widehat{S E}$ is the estimated $\mathrm{SE}$ of the sampling distribution for $\hat{\mu}$.

We calculated 2 additional intervals to convey the estimated spread of each random-effects distribution. The first was a $95 \%$ population effects interval (PEI), 95\% PEI $=\hat{\mu} \pm 1.96 \tau$, where $\tau$ is the restricted maximum likelihood estimate of the SD of the random-effects distribution. The 95\% $\mathrm{PEI}$ is the central range within which $95 \%$ of populations' RD values are estimated to lie..$^{50,51}$ The second was a $95 \%$ prediction interval (PI),

$$
95 \% \mathrm{PI}=\hat{\mu} \pm t_{k-2} \sqrt{\tau^{2}+\widehat{S E}^{2}},
$$

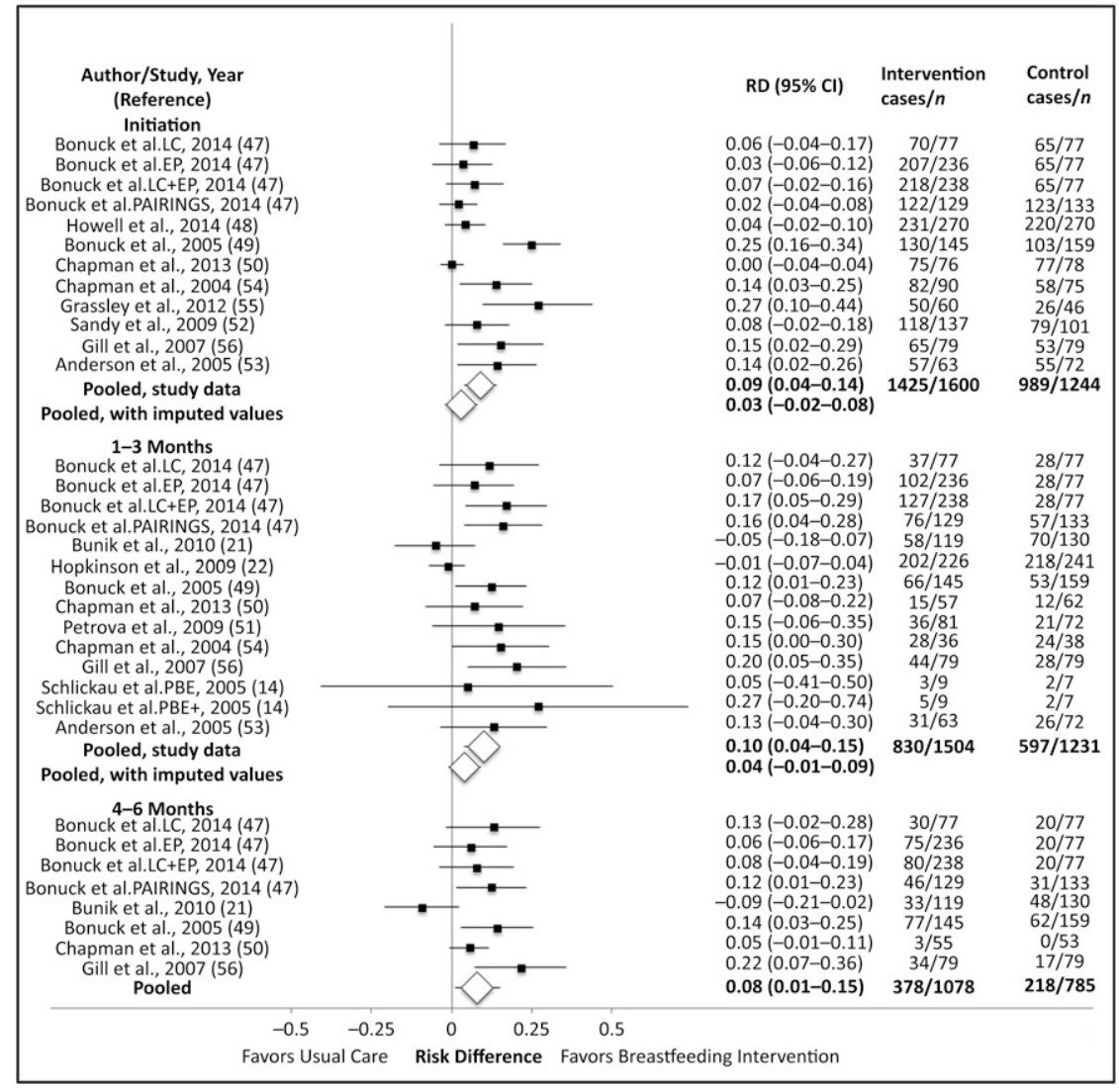

FIGURE 2

Summary RDs of breastfeeding interventions for any breastfeeding versus no breastfeeding

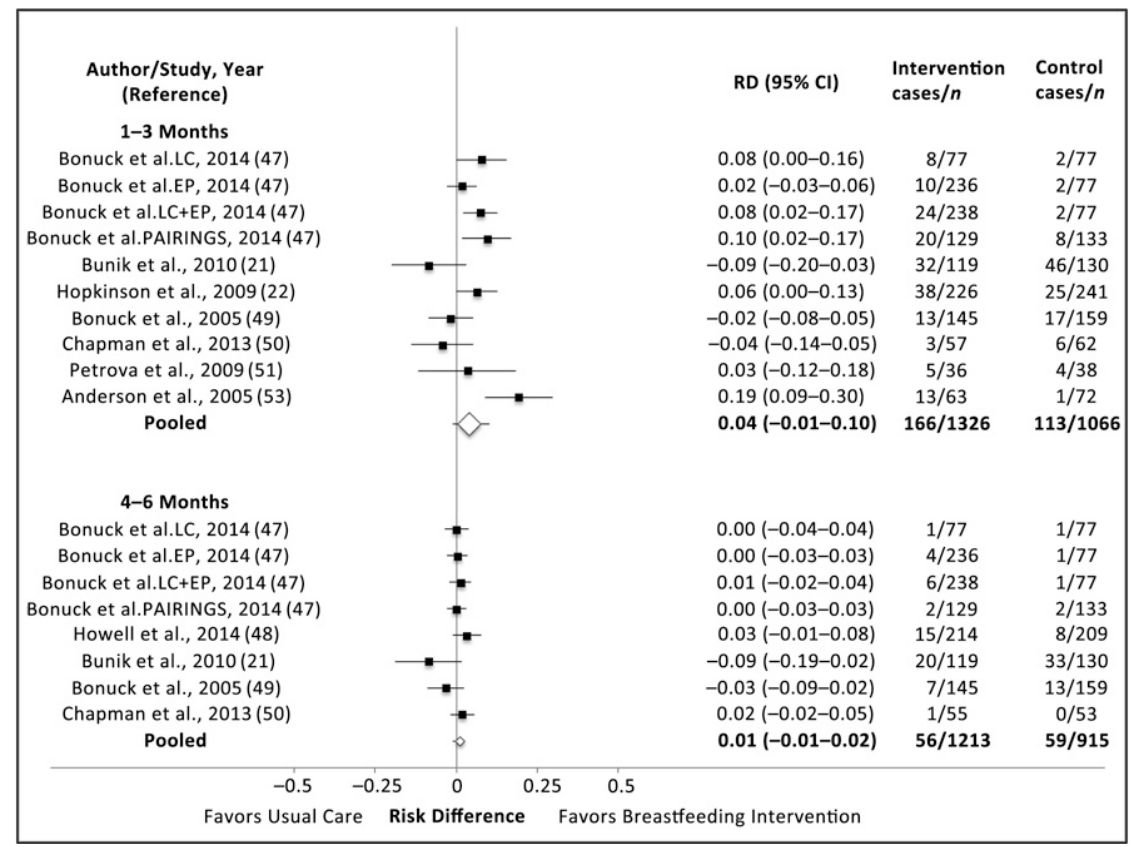

FIGURE 3

Summary RDs of breastfeeding interventions for exclusive breastfeeding versus nonexclusive breastfeeding. 
TABLE 2 Meta-analysis Results of Trials of the Association Between Breastfeeding Interventions and Any or Exclusive Breastfeeding Outcomes

\begin{tabular}{|c|c|c|c|c|c|c|}
\hline Estimate & $\begin{array}{c}\text { Number of } \\
\text { Estimates }(k)\end{array}$ & $\begin{array}{l}\text { Cochran } Q \text { ( } P \\
\text { value) }\end{array}$ & $\begin{array}{l}\text { Random-effects } \\
\text { variance }\left(\tau^{2}\right)\end{array}$ & $R D_{R E}^{\mathrm{a}}(95 \% \mathrm{Cl})$ & $\begin{array}{l}\text { 95\% Population } \\
\text { Effects Interval }\end{array}$ & $\begin{array}{l}\text { 95\% Prediction } \\
\text { Interval }\end{array}$ \\
\hline Initiation, study data & 12 & $41.32(<.001)$ & 0.005 & 0.09 (0.04 to 0.14$)$ & $(-0.04$ to 0.22$)$ & $(-0.07$ to 0.25$)$ \\
\hline $\begin{array}{l}\text { Initiation, with imputed } \\
\text { values }\end{array}$ & 18 & $96.4(<.001)$ & 0.009 & $0.03(-0.02$ to 0.08$)$ & $(-0.20$ to 0.26$)$ & $(-0.22$ to 0.28$)$ \\
\hline $\begin{array}{l}\text { 1- to 3-mo any } \\
\text { breastfeeding, study } \\
\text { data }\end{array}$ & 14 & $23.75(.03)$ & 0.004 & 0.10 (0.04 to 0.15$)$ & $(-0.02$ to 0.21$)$ & $(-0.05$ to 0.24$)$ \\
\hline $\begin{array}{l}\text { 1- to 3-mo any } \\
\text { breastfeeding, with } \\
\text { imputed values }\end{array}$ & 20 & $47.98(<.001)$ & 0.007 & $0.04(-0.01$ to 0.09$)$ & $(-0.14$ to 0.22$)$ & $(-0.15$ to 0.23$)$ \\
\hline $\begin{array}{l}\text { 4- to 6-mo any } \\
\text { breastfeeding }\end{array}$ & 8 & $14.95(.04)$ & 0.004 & $0.08(0.01$ to 0.15$)$ & $(-0.04$ to 0.20$)$ & $(-0.08$ to 0.25$)$ \\
\hline $\begin{array}{l}\text { 1- to } 3 \text {-mo exclusive } \\
\text { breastfeeding }\end{array}$ & 10 & $25.23(.003)$ & 0.003 & 0.04 ( -0.01 to 0.10$)$ & $(-0.06$ to 0.15$)$ & $(-0.09$ to 0.18$)$ \\
\hline $\begin{array}{l}\text { 4- to } 6 \text {-mo exclusive } \\
\text { breastfeeding }\end{array}$ & 8 & $7.40(.39)$ & 0 & $0.01(-0.01$ to 0.02$)$ & $(0.01$ to 0.01$)$ & $(-0.01$ to 0.02$)$ \\
\hline
\end{tabular}

a Random-effects summary RD.

where $k$ is the number of RD estimates in the meta-analysis and $\tau_{k-2}$ is the 97.5th percentile of a $t$ distribution with $k-2$ degrees of freedom. In hypothetical repetitions of the entire literature-generating process, 95\% of the 95\% PIs will cover the true RD in a future study population. Hence, a 95\% PI may be informally interpreted as a 95\% CI for the RD in the "next" study population. ${ }^{52-54}$ Overall heterogeneity was assessed for each outcome by calculating a $P$ value for the Cochran $Q$ statistic. A funnel plot was examined visually for asymmetry and statistically by the tests of Egger et al. ${ }^{55}$ and Begg and Mazumdar, ${ }^{56}$ as well as by the trim-and-fill method of Duval and Tweedie $^{57}$ (Supplemental Figs 4 and 8).

The univariable meta-regression model was used to explore heterogeneity of random-effects estimates by 3 potentially influential and clinically meaningful intervention characteristics: timing of the intervention (prenatal, postpartum, or combined prenatal and postpartum), intervention intensity (number of intended contacts), and provider delivering the intervention (medical provider, International Board Certified Lactation Consultant [IBCLC], or lay provider) (Table 3). Univariable meta-regression was also conducted for 3 study characteristics that might affect random-effects estimates: publication year (2010 to 2014 vs 2004 to 2009 , since population-level breastfeeding estimates have changed over time), study design (randomized controlled trial [RCT] with allocation concealed or bias minimized, RCT with no allocation concealment, or non-RCT), and breastfeeding intention inclusion criterion (Supplemental Table 5). The small number of trials and clustering of study characteristics prevented our fitting multivariable meta-regression models. Intercooled Stata (version 11, Stata Corp., College Station, TX) was used for these analyses.

\section{RESULTS}

Our initial database search yielded 321 nonduplicate citations (Fig 1). Review of these titles yielded 301 potentially eligible abstracts. From these abstracts, 20 publications met inclusion criteria for full-text review, from which 14 studies describing 17 interventions met inclusion criteria for this systematic review and metaanalysis. ${ }^{14,21,22,38-47}$ References from publications selected for full-text review and from a recent review of breastfeeding interventions for minority women ${ }^{28}$ yielded no additional publications meeting eligibility criteria.

Six of the 14 included studies were rated as good quality, ${ }^{21,22,38-40} 3$ as fair, ${ }^{41-43}$ and 5 as poor ${ }^{14,44-47}$ (quality criteria provided in Supplemental Table 4). Eleven of the 14 studies were RCTs $21,22,38-43,45,47$; however, 2 of these analyzed only a subgroup of participants owing to application of postrandomization inclusion criteria, which may have minimized the benefits of randomization. ${ }^{43,45}$ of the 12 RCTs, 4 concealed allocation assignment from study staff during outcome assessment ${ }^{22,38,39}$ and 3 minimized bias by interviewing mothers about intervention contact only after collecting breastfeeding outcome data. ${ }^{41,43,47}$

\section{Study Characteristics}

Table 1 summarizes study characteristics. The 14 included studies were published between 2004 and 2014. All 14 were prospective, controlled studies of a single or multiple-armed breastfeeding intervention initiated in a health care setting and conducted in a majority Latina population. Sample sizes ranged from 30 to 666 , totaling 4000 participants overall. Study populations ranged from $56 \%$ to $100 \%$ Latina.

Populations varied by place of birth, 
TABLE 3 Meta-regression Results of Trials of the Association Between Breastfeeding Interventions and Breastfeeding Outcomes by Potentially Influential Intervention Characteristics

\begin{tabular}{|c|c|c|c|c|c|c|c|c|c|}
\hline \multirow[t]{2}{*}{ Study Characteristic } & \multicolumn{3}{|c|}{ Initiation } & \multicolumn{3}{|c|}{ 1-3 Months } & \multicolumn{3}{|c|}{ 4-6 Months } \\
\hline & Study $n$ & $P$ & $\mathrm{RD}(95 \% \mathrm{Cl})$ & $\begin{array}{c}\text { Study } \\
n\end{array}$ & $P$ & RD $(95 \% \mathrm{Cl})$ & $\begin{array}{l}\text { Study } \\
n\end{array}$ & $P$ & $\mathrm{RD}(95 \% \mathrm{Cl})$ \\
\hline $\begin{array}{l}\text { Any breastfeeding, overall } \\
\text { Timing of intervention }\end{array}$ & 12 & $<.001$ & 0.09 (0.04 to 0.14$)$ & 14 & .03 & 0.10 (0.04 to 0.15$)$ & 8 & .037 & 0.08 (0.01 to 0.15$)$ \\
\hline $\begin{array}{l}\text { Prenatal and } \\
\text { postpartum }\end{array}$ & 10 & $<.001$ & 0.09 (0.03 to 0.15$)$ & 10 & .96 & 0.13 (0.08 to 0.18 ) & 7 & .41 & 0.10 (0.04 to 0.15$)$ \\
\hline Postpartum & 2 & .02 & $0.12(-0.03$ to 0.26$)$ & 2 & .56 & $-0.02(-0.07$ to 0.04$)$ & 1 & - & $-0.09(-0.25$ to 0.06$)$ \\
\hline Prenatal & 0 & & - & 2 & .5 & $0.16(-0.21$ to 0.52$)$ & 0 & - & - \\
\hline \multicolumn{10}{|l|}{ Intervention intensity } \\
\hline$>6$ contacts & 6 & .05 & $0.06(-0.01$ to 0.14$)$ & 6 & .08 & 0.11 (0.04 to 0.18 ) & 5 & .01 & $0.07(-0.03$ to 0.17$)$ \\
\hline $3-6$ contacts & 5 & .004 & 0.14 (0.05 to 0.23$)$ & 5 & .93 & $0.12(0.04$ to 0.20$)$ & 3 & .56 & $0.11(-0.03$ to 0.24$)$ \\
\hline $1-2$ contacts & 1 & - & $0.04(-0.12$ to 0.20$)$ & 3 & .5 & $0.00(-0.10$ to 0.10$)$ & 0 & - & - \\
\hline \multicolumn{10}{|l|}{ Provider } \\
\hline Medical provider & 5 & .11 & $0.06(-0.02$ to 0.14$)$ & 6 & .13 & 0.06 ( -0.03 to 0.15$)$ & 4 & .05 & $0.04(-0.06$ to 0.14$)$ \\
\hline IBCLC & 3 & .03 & $0.16(0.05$ to 0.27$)$ & 4 & .84 & 0.14 (0.04 to 0.25$)$ & 3 & .65 & $0.16(0.03$ to 0.29$)$ \\
\hline Lay provider & 4 & .02 & $0.07(-0.02$ to 0.16$)$ & 4 & .10 & $0.13(-0.09$ to 0.35$)$ & 1 & - & $0.05(-0.10$ to 0.21$)$ \\
\hline $\begin{array}{l}\text { Exclusive breastfeeding, } \\
\text { overall }\end{array}$ & & & & 10 & .003 & 0.04 (0.00 to 0.08$)$ & 8 & .39 & $0.01(-0.01$ to 0.02$)$ \\
\hline \multicolumn{10}{|l|}{ Timing of intervention } \\
\hline $\begin{array}{l}\text { Prenatal and } \\
\text { postpartum }\end{array}$ & - & - & - & 8 & .01 & 0.05 (-0.01 to 0.11$)$ & 6 & .74 & $0.00(-0.02$ to 0.02$)$ \\
\hline Postpartum & - & - & - & 2 & .03 & $0.01(-0.12$ to 0.13$)$ & 2 & .04 & $0.01(-0.04$ to 0.07$)$ \\
\hline Prenatal & - & - & - & 0 & - & - & 0 & - & - \\
\hline \multicolumn{10}{|l|}{ Intervention intensity } \\
\hline$>6$ contacts & - & - & - & 5 & .001 & 0.05 (-0.04 to 0.14$)$ & 4 & .27 & $0.01(-0.02$ to 0.03$)$ \\
\hline $3-6$ contacts & - & - & - & 4 & .33 & $0.03(-0.07$ to 0.12$)$ & 3 & .49 & $0.00(-0.03$ to 0.03$)$ \\
\hline $1-2$ contacts & - & - & - & 1 & - & $0.06(-0.12$ to 0.25$)$ & 1 & - & $0.03(-0.03$ to 0.09$)$ \\
\hline \multicolumn{10}{|l|}{ Provider } \\
\hline Medical provider & - & - & - & 4 & .02 & 0.03 (-0.06 to 0.13$)$ & 5 & .30 & $0.01(-0.02$ to 0.03$)$ \\
\hline IBCLC & - & - & - & 3 & .19 & $0.03(-0.09$ to 0.15$)$ & 2 & .32 & -0.01 ( -0.05 to 0.03$)$ \\
\hline Lay provider & - & - & - & 3 & .004 & 0.07 (-0.05 to 0.18$)$ & 1 & - & 0.02 (-0.03 to 0.07$)$ \\
\hline
\end{tabular}

years of US residence, and language preference, considered proxies for acculturation. ${ }^{58}$ Approximately $70 \%$ of studies targeted lowincome populations. Although only 2 studies enrolled primiparous women exclusively, ${ }^{14,21}$ almost half of participants in most studies were first-time mothers. One study recruited only overweight or obese participants. ${ }^{41}$ Six studies required that participants consider or intend to breastfeed. ${ }^{21,22,41,43,44,47}$ Ten studies recruited from ambulatory prenatal care settings, $14,38,40-43,45-47$ and 4 recruited from labor and delivery units. ${ }^{21,22,39,44}$ Definitions of usual care ranged from no explicit breastfeeding support to BabyFriendly Hospital standard of care.

Outcome measures were heterogeneously defined. Most studies measured prevalence of any breastfeeding at varying time points as a proxy for breastfeeding duration. Only 4 studies reported true breastfeeding duration since birth. ${ }^{39,40,46,47}$ Seven studies measured prevalence of exclusive breastfeeding, 14,22,38,41,42,45 and 1 measured "predominant" breastfeeding, defined as $\leq 4 \mathrm{oz}$ formula per day, as no participants exclusively breastfed. ${ }^{21}$

\section{Intervention Characteristics}

Intervention components included breastfeeding support and education delivered in person or by telephone. Seven interventions used phone calls, ${ }^{21,22,39,40,42,43,46} 7$ used optional or required home visits, ${ }^{38,40,43,45-47}$ and 13 used clinic or in-hospital visits to provide interpersonal support. ${ }^{14,22,38-47}$ Only 1 intervention provided breastfeeding education without an interpersonal support component. ${ }^{38}$ Intervention intensity, defined as the number of intended patient contacts, ranged from 1 to 14 , with 1 intervention contacting women weekly until they weaned. ${ }^{40}$ Nine interventions involved both prenatal and postpartum points of contact, ${ }^{38,40-43,45,46} 2$ were initiated in-hospital after delivery, ${ }^{39,44} 2$ were initiated in the early postpartum period, ${ }^{21,22}$ and 1 included prenatal contact alone. ${ }^{14}$ Duration of follow-up ranged from 1 week to 1 year. Five interventions formally sought any degree of family involvement in the intervention. ${ }^{38,41,45,47}$ All but 1 intervention employed bilingual and/ or bicultural staff members and used bilingual materials ${ }^{44}$; additionally, 6 interventions explicitly addressed Latina-specific cultural and social factors in their protocols. ${ }^{14,21,39,41,43,46}$ 
Interventions were delivered by IBCLCs, ${ }^{38,40,42,46}$ physicians, ${ }^{38}$ nurses, ${ }^{14,21,44}$ social workers, ${ }^{39}$ or lay providers. ${ }^{22,41,43,45,47}$ Interventions using lay providers formally trained peers drawn from the same communities as study subjects, requiring that lay providers have breastfed $\geq 6$ months, ${ }^{41,43,47}$ have worked $\geq 1$ year in a Special Supplemental Program for Women, Infants, and Children (WIC) clinic, $^{22}$ or become certified WIC peer counselors. ${ }^{45}$ All 5 interventions with significant increases in any breastfeeding at 1 to 3 months and 4 to 6 months included prenatal and postpartum components delivered by an IBCLC. ${ }^{38,40,46}$ of these, 4 were rated as good quality 38,40 and 1 as poor quality. ${ }^{46}$ The 3 interventions with significant increases in exclusive breastfeeding at 1 to 3 months also included both prenatal and postpartum components and $>6$ intended points of contact with participants. ${ }^{38,47}$ All interventions that significantly increased any or exclusive breastfeeding at 1 to 3 months or any breastfeeding at 4 to 6 months involved $\geq 1$ visit by a provider to the participant's home. ${ }^{38,40,43,46,47}$

\section{Meta-analyses}

\section{Crude Analyses}

From the 17 interventions described in the 14 included studies, 34 RDs were calculated to estimate the effect of breastfeeding interventions on the risk of any breastfeeding versus no breastfeeding across the 3 time intervals (Fig 2). Cochrane $Q$ statistics indicated evidence of heterogeneity among trial-specific effect estimates for all 3 summary estimates $(P<.05)$ (Table 2$)$. For both initiation and 1- to 3-month any breastfeeding estimates, there was visual appearance of asymmetry in the funnel plots, reinforced by Egger et al. ${ }^{55}$ and Begg and Mazumdar ${ }^{56}$ tests for small-study effects indicating evidence of publication bias (Supplemental Figs 4 and 5). A trim-and-fill analysis imputed 6 possibly missing trial results for initiation, reducing the randomeffects summary estimate from 0.09 to 0.03 (95\% CI -0.02 to $0.08,95 \%$ PEI -0.20 to $0.26,95 \%$ PI -0.22 to 0.28 ) with an NNT of 37 . For 1- to 3-month any breastfeeding, 6 possibly missing trial results were imputed, reducing the random-effects summary estimate from 0.10 to 0.04 (95\% CI -0.01 to $0.09,95 \%$ PEI -0.14 to $0.22,95 \%$ PI -0.15 to 0.23 ) with an NNT of 24. No evidence of publication bias was found for 4- to 6-month estimates, which resulted in a pooled RD of 0.08 (95\% CI 0.01 to $0.15 ; 95 \% \mathrm{PEI}-0.04$ to $0.20 ; 95 \%$ PI -0.08 to 0.25 ) with an NNT of 12 (Supplemental Fig 6).

Eleven of the 17 interventions described above reported the effect of interventions on risk of exclusive breastfeeding versus nonexclusive breastfeeding, providing 18 RD estimates (Fig 3). The estimated RD for 1 to 3 months was 0.04 (95\% CI -0.01 to 0.10 ; $95 \%$ PEI -0.06 to 0.15 ; $95 \%$ PI -0.09 to 0.18 ) and for 4 to 6 months was 0.01 (95\% CI -0.01 to 0.02 ; $95 \%$ PEI 0.01 to 0.01 ; $95 \%$ PI -0.01 to 0.02 ), resulting in NNTs of 23 and 199, respectively. Cochrane $Q$ statistics indicated evidence of heterogeneity only for 1- to 3-month estimates (Table 2). There was no visual evidence of heterogeneity for either estimate, and neither funnel plots nor Egger et al. ${ }^{55}$ and Begg and Mazumdar ${ }^{56}$ tests indicated evidence of bias (Supplemental Figs 7 and 8).

\section{Univariable Meta-regression}

Table 3 describes univariable meta-regression results for any breastfeeding and exclusive breastfeeding by strata of clinically relevant intervention characteristics. For any breastfeeding and exclusive breastfeeding at 1 to 3 months and any breastfeeding at 4 to 6 months, interventions with both prenatal and postpartum contact resulted in larger effect estimates than interventions using postpartum contact alone. Across all time intervals, moderate intervention intensity (defined as 3 to 6 patient contacts) and delivery by an IBCLC showed slightly larger estimates of effect on any breastfeeding versus no breastfeeding. Additionally, interventions delivered by lay providers showed slightly larger estimates of effect on any breastfeeding than interventions delivered by medical providers, and the effect of lay providers was stronger than both IBCLCs and medical providers for exclusive breastfeeding at 1 to 3 months. Because of the small sample size and low overall estimate of effect, the meta-regression results for exclusive breastfeeding did not vary significantly by intervention characteristics.

Supplemental Table 5 describes meta-regression results by study characteristics. Compared with initiation or 1- to 3-month any breastfeeding estimates, 4- to 6-month any breastfeeding estimates and exclusive breastfeeding estimates at either time interval were more likely to be drawn from recent studies with $>200$ participants and a randomized study design. These study characteristics were all generally associated with smaller effect estimates. Studies reporting exclusive breastfeeding estimates were also more likely to have a breastfeeding intention inclusion criterion compared with studies reporting any breastfeeding estimates.

\section{DISCUSSION}

Clinical breastfeeding interventions targeting Latinas appear to increase any breastfeeding and exclusive breastfeeding at varying time points. Although random-effects summary estimates for initiation and 1- to 3-month any breastfeeding were 
attenuated and CIs crossed the null after imputation to correct for publication bias, all random-effects summary estimates consistently favored clinical breastfeeding interventions over usual care. However, the published evidence for Latinas is limited, and studies have varying methodologic rigor.

The small magnitude of summary estimates and the substantial heterogeneity across studies are consistent with findings from previous meta-analyses of breastfeeding interventions. Guise et al., ${ }^{26}$ Chung et al, ${ }^{27}$ and Renfrew et $\mathrm{al}^{59}$ found breastfeeding interventions to be beneficial in the general population, and Ibanez et al $^{60}$ identified benefits specifically among low-income women. These reviews also observed high levels of heterogeneity across intervention characteristics and effect estimates, but similarly concluded that breastfeeding interventions appear more effective than usual care for increasing duration of any breastfeeding. ${ }^{26,27,59,60}$ Unlike previous systematic reviews, our review estimated smaller effects for exclusive breastfeeding, possibly due to a higher prevalence of mixed feeding among Latinas. Given the low exclusive breastfeeding rates in Latina populations, future interventions should aim to reduce barriers to exclusive breastfeeding specific to Latinas, addressing selfefficacy, family and social support, and psychosocial factors. ${ }^{10,61}$

Whereas strong evidence of publication bias was observed for the literature reporting initiation and 1- to 3-month any breastfeeding estimates, such bias was not observed among studies reporting exclusive breastfeeding or 4- to 6-month any breastfeeding outcomes. These findings are plausible given the generally weaker methodologic rigor of studies reporting shorterterm outcomes, where authors may have been likely to publish statistically significant results and disregard nonsignificant findings without a major incursion of time and funding. On the other hand, studies either following women longer or aiming to increase breastfeeding exclusivity may have been more likely to report both significant and nonsignificant outcomes owing to their generally larger study sizes and stronger designs, which require more substantial investments. Future studies should report and publish all findings, including null results. Despite the evidence of publication bias for initiation and 1- to 3-month any breastfeeding, effect estimates at each time interval indicate a positive effect of interventions on breastfeeding outcomes among Latinas.

In examining potential methodologic and etiologic factors that modify these effects, we observed that the diversity of study populations, intervention and "usual care" standards, and breastfeeding outcome measures contributed to heterogeneous estimates. RCTs and studies with larger sample sizes produced smaller effect estimates than less rigorous and smaller studies, likely owing to minimization of selection bias and other confounding factors across trial arms. For any breastfeeding at 1 to 3 and 4 to 6 months, interventions with prenatal and postpartum components produced larger effects than interventions targeting only the postpartum period, indicating the importance of providing breastfeeding support during both critical periods. Moderate intervention intensity, defined as 3 to 6 contacts between provider and mother, was associated with larger effects on any breastfeeding than either less or more frequent contact, highlighting the positive effect of only moderately timeintensive breastfeeding interventions and suggesting that highly resource-intense interventions may not be necessary to achieve maximal benefit.

Interventions delivered by IBCLCs were associated with the largest effects on any breastfeeding across time intervals, and interventions delivered by lay providers were associated with stronger effects than both IBCLCs and medical providers for exclusive breastfeeding at 1 to 3 months. With the former U.S. Surgeon General's call for integrated lactation support in primary care settings, including improved access to IBCLCs, ${ }^{62}$ these findings reiterate the importance of including affordable and accessible IBCLC and peer counselor services in a variety of clinical settings serving Latinas. We reiterate the recommendation by Chung et $\mathrm{al}^{27}$ for future studies to directly compare providers, including medical providers, IBCLCs, and lay providers, considering both the time and cost associated with these breastfeeding intervention delivery models.

Our review has several strengths, such as the large number of subjects $(n=4000)$ from included studies. Furthermore, all included interventions had comparison groups. Although definitions of breastfeeding interventions and "usual care" varied substantially, in some cases biasing the intervention effect toward the null, these diverse contexts improve generalizability to a wide range of clinical settings and delivery formats. The variety of intervention types and intensities allowed us to identify the most effective interventions as those with moderate intensity, IBCLC or lay providers, prenatal and postpartum components, and a home visit. This suggests that future breastfeeding interventions targeting Latina women should ideally begin in the prenatal setting, involving frequent contact with an IBCLC or lay provider. 
Our review was limited by evidence of publication bias among studies reporting initiation and any breastfeeding at 1 to 3 months, resulting in possibly skewed meta-regression results for which data could not be imputed. Our conclusions are further limited by the internal validity of the studies reviewed; common flaws included failure to maintain comparable groups, inadequate allocation concealment, and poor adherence to the intent-to-treat principle. Additionally, our use of the term Latina may have masked important subgroup differences that could contribute to the heterogeneity in effect estimates. The aggregation of diverse ethnic subgroups and the limited data on potentially confounding cultural and medical factors, such as acculturation, language, and family support, reduced our ability to identify homogeneous intervention effects across study populations. Intervention effects were drawn from study populations with varying proportions of Latina women, limiting our ability to draw population-specific conclusions; future studies should be conducted exclusively among Latinas while accounting for subgroup variations, such as acculturation level and immigrant status. Investigators should consider how multidimensional components of acculturation directly or indirectly influence breastfeeding outcomes within and between Latina subgroups. Where study populations are heterogeneous, subgroup analyses by race/ethnicity should be presented, as Anderson et $\mathrm{al}^{48}$ report for their peer counseling intervention, to compare the effect of interventions delivered to women who may share similar barriers. This will require that researchers ensure adequate sample sizes of Latina subgroups to permit detection of stratum-specific statistical associations.

Breastfeeding interventions targeting Latina populations were identified only in studies published since 2004, highlighting a need for continued research. The USPSTF recently proposed a new research plan to review primary care breastfeeding interventions with an explicit focus on variations in effectiveness by racial/ethnic population subgroups. ${ }^{63}$ This provides an opportunity for future trials to be conducted within homogeneous Latina subgroups, such as women with similar acculturation status and country of origin. Future studies would also benefit from uniformity in defining breastfeeding outcomes, measuring breastfeeding since birth to permit calculation of rates, and reporting outcomes at consistent time points to facilitate comparison across studies. Finally, although it is difficult to blind participants and intervention staff in breastfeeding trials, more robust allocation concealment procedures are needed for study staff to avoid measurement bias. By improving the methodological rigor of interventions, a more accurate estimation of their effect on breastfeeding duration and exclusivity could be obtained.

\section{CONCLUSIONS}

Available evidence suggests a favorable effect of clinical interventions on any breastfeeding and exclusive breastfeeding among Latinas. Strong evidence indicates that improved breastfeeding outcomes benefit both infant and maternal health, and the potential for harm from breastfeeding promotion interventions is low. Continued clinical and policy support is necessary to help Latina mothers achieve their breastfeeding goals and bring population-level breastfeeding recommendations within reach.

\section{ACKNOWLEDGMENTS}

The authors thank Anthony J. Viera, MD, MPH, and Sandra Martin, PhD, for their editorial support on early drafts of this review.

\section{ABBREVIATIONS}

CI: confidence interval

IBCLC: international boardcertified lactation consultant

NNT: number needed to treat

PEI: population effects interval

PI: prediction interval

RCT: randomized controlled trial

RD: risk difference

WIC: Special Supplemental Program for Women, Infants, and Children

\footnotetext{
Ms Wouk led data analyses and interpretation of data; Ms Wouk and Dr Lara-Cinisomo approved the final manuscript as submitted; Ms Wouk and Dr McKenney conceptualized and conducted the systematic review (performing search strategies, screening articles, and abstracting data) and drafted the initial manuscript; Ms. Wouk and Drs McKenney, Poole, Petrick, and Lara-Cinisomo reviewed and revised the manuscript; Dr Stuebe provided subject matter expertise for interpreting the clinical significance of breastfeeding interventions and reviewed and revised the manuscript with attention to substantive breastfeeding issues; Drs Poole and Petrick supervised the lead author in meta-analytic methods, advising on presentation of the data; and Dr Lara-Cinisomo assisted with the initial conception of the work, including identifying variables for the meta-analysis and operationalizing the research question, and supervised the conduct of the metaanalysis and systematic review.
}

DOI: 10.1542/peds.2015-2423

Accepted for publication 0ct 5, 2015 
Address correspondence to Kathryn Wouk, Carolina Global Breastfeeding Institute, Department of Maternal and Child Health, Gillings School of Global Public Health, University of North Carolina at Chapel Hill, CB\#7445, Chapel Hill, NC 27599. E-mail: khouk@live.unc.edu

PEDIATRICS (ISSN Numbers: Print, 0031-4005; Online, 1098-4275).

Copyright (C) 2016 by the American Academy of Pediatrics

FINANCIAL DISCLOSURE: The authors have indicated they have no financial relationships relevant to this article to disclose.

FUNDING: This meta-analysis was supported in part by the Carolina Global Breastfeeding Institute, the National Institutes of Health (MH093315, UL1TR000083, KL2TR000084, and TL1TR000085), the National Cancer Institute Intramural Research Program, the Foundation of Hope for Research and Treatment of Mental IIIness, and the North Carolina Translational and Clinical Sciences Institute. Funded by the National Institutes of Health (NIH).

POTENTIAL CONFLICT OF INTEREST: The authors have indicated they have no potential conflicts of interest to disclose.

\section{REFERENCES}

1. Ip S, Chung M, Raman G, et al. Breastfeeding and maternal and infant health outcomes in developed countries. Evid Rep Technol Assess (Full Rep). 2007;153:1-186

2. Breastfeeding and the Use of Human Milk: Section on Breastfeeding. Pediatrics. 2012;129(3). Available at: www.pediatrics.org/cgi/content/full/ 129/3/e827

3. American College of Obstetricians and Gynecologists. Breastfeeding: Maternal and Infant Aspects. 2007. Available at www.acog.org/-/media/CommitteeOpinions/Committee-on-Health-Carefor-Underserved-Women/co361.pdf? $\mathrm{dmc}=1 \& \mathrm{ts}=20151014 \mathrm{~T} 1459406499$. Accessed October 14, 2015

4. American Academy of Family Physicians. Family Physicians Supporting Breastfeeding (Position Paper). 2008. Available at: www.aafp. org/about/policies/all/breastfeedingsupport.html. Accessed January 22, 2015

5. Bartick M, Reinhold A. The burden of suboptimal breastfeeding in the United States: a pediatric cost analysis. Pediatrics. 2010;125(5). Available at: www.pediatrics.org/cgi/content/full/ 125/5/e1048

6. Bartick MC, Stuebe AM, Schwarz EB, Luongo C, Reinhold AG, Foster EM. Cost analysis of maternal disease associated with suboptimal breastfeeding. Obstet Gynecol. 2013;122(1):111-119

7. U.S. Department of Health and Human Services. Healthy People 2020: Maternal, Infant, and Child Health. Available at: www.healthypeople.gov/ 2020/topics-objectives/topic/maternalinfant-and-child-health. Accessed March 30, 2015
8. Breastfeeding among U.S. children born 2001-2011, CDC National Immunization Survey. 2014. Available at: www.cdc.gov/breastfeeding/data/ nis_data/. Accessed January 22, 2015

9. Centers for Disease Control and Prevention: National Center for Chronic Disease Prevention and Health Promotion. Rates of Any and Exclusive Breastfeeding by Sociodemographics Among Children Born in 2012. 2015. Available at: www.cdc.gov/ breastfeeding/data/nis_data/ratesany-exclusive-bf-socio-dem-2011.htm. Accessed October 14, 2015

10. Gill SL. Breastfeeding by Hispanic women. J Obstet Gynecol Neonatal Nurs. 2009;38(2):244-252

11. McCann MF, Baydar N, Williams RL. Breastfeeding attitudes and reported problems in a national sample of WIC participants. J Hum Lact. 2007;23(4):314-324

12. Li R, Fein SB, Chen J, Grummer-Strawn LM. Why mothers stop breastfeeding: mothers' self-reported reasons for stopping during the first year. Pediatrics. 2008;122(suppl 2):S69-S76

13. Hurley KM, Black MM, Papas MA, Quigg AM. Variation in breastfeeding behaviours, perceptions, and experiences by race/ethnicity among a low-income statewide sample of Special Supplemental Nutrition Program for Women, Infants, and Children (WIC) participants in the United States. Matern Child Nutr. 2008;4(2):95-105

14. Schlickau J, Wilson M. Development and testing of a prenatal breastfeeding education intervention for Hispanic women. J Perinat Educ. 2005;14(4):24-35
15. Howell EA, Mora PA, Horowitz CR, Leventhal H. Racial and ethnic differences in factors associated with early postpartum depressive symptoms. Obstet Gynecol. 2005;105(6):1442-1450

16. Liu CH, Tronick E. Prevalence and predictors of maternal postpartum depressed mood and anhedonia by race and ethnicity. Epidemiol Psychiatr Sci. 2013;23(2):1-9

17. Dennis CL, McQueen K. The relationship between infant-feeding outcomes and postpartum depression: a qualitative systematic review. Pediatrics. $2009 ; 123$ (4). Available at: www pediatrics.org/cgi/content/full/123/4/ e736

18. Dias CC, Figueiredo B. Breastfeeding and depression: a systematic review of the literature. J Affect Disord. 2015;171:142-154

19. Gress-Smith JL, Luecken LJ, LemeryChalfant K, Howe R. Postpartum depression prevalence and impact on infant health, weight, and sleep in lowincome and ethnic minority women and infants. Matern Child Health J. 2012;16(4):887-893

20. Waldrop J. Exploration of reasons for feeding choices in Hispanic mothers. MCN Am J Matern Child Nurs. 2013;38(5):282-288

21. Bunik M, Shobe P, O'Connor ME, et al. Are 2 weeks of daily breastfeeding support insufficient to overcome the influences of formula? Acad Pediatr. 2010;10(1):21-28

22. Hopkinson J, Konefal Gallagher M. Assignment to a hospital-based breastfeeding clinic and exclusive breastfeeding among immigrant Hispanic mothers: a randomized, 
controlled trial. J Hum Lact. 2009;25(3):287-296

23. Denman-Vitale S, Murillo EK. Effective promotion of breastfeeding among Latin American women newly immigrated to the United States. Holist Nurs Pract. 1999;13(4):51-60

24. Rassin DK, Markides KS, Baranowski T, Richardson CJ, Mikrut WD, Bee $D E$. Acculturation and the initiation of breastfeeding. J Clin Epidemiol. 1994:47(7):739-746

25. Centers for Disease Control and Prevention. The CDC Guide to Strategies to Support Breastfeeding Mothers and Babies. 2013. Available at: www.cdc.gov/breastfeeding/pdf/BFGuide-508.PDF. Accessed June 18, 2015.

26. Guise JM, Palda V, Westhoff C, Chan BK, Helfand M, Lieu TA; U.S. Preventive Services Task Force. The effectiveness of primary care-based interventions to promote breastfeeding: systematic evidence review and meta-analysis for the US Preventive Services Task Force. Ann Fam Med. 2003;1 (2):70-78

27. Chung M, Raman G, Trikalinos T, Lau J, Ip S. Interventions in primary care to promote breastfeeding: an evidence review for the U.S. Preventive Services Task Force. Ann Intern Med. 2008;149(8):565-582

28. Chapman DJ, Pérez-Escamilla $R$. Breastfeeding among minority women: moving from risk factors to interventions. Adv Nutr. 2012;3(1):95-104

29. Ennis SR, Ríos-Vargas M, Albert NG. The Hispanic Population: 2010. 2010 U.S. Census Brief. Washington, DC: US Census Bureau; 2011. Available at: www.census.gov/prod/cen2010/briefs/ c2010br-04.pdf. Accessed 0ctober 14, 2015

30. DeNavas-Walt C, Proctor BD, Smith JC. Income, Poverty and Health Insurance Coverage in the United States: 2010. 2011:1-87. Available at: www.census. gov/prod/2011pubs/p60-239.pdf. Accessed June 18, 2015

31. Carter-Pokras 0D, Gergen PJ. Reported asthma among Puerto Rican, MexicanAmerican, and Cuban children, 1982 through 1984. Am J Public Health 1993;83(4):580-582
32. Findley S, Lawler K, Bindra M, Maggio L, Penachio MM, Maylahn C. Elevated asthma and indoor environmental exposures among Puerto Rican children of East Harlem. J Asthma. 2003;40(5):557-569

33. Gupta RS, Bewtra M, Prosser LA, Finkelstein JA. Predictors of hospital charges for children admitted with asthma. Ambul Pediatr. 6(1):15-20

34. Beckles GL, Chou C-F. Diabetes-United States, 2006 and 2010. 2013. Available at: www.cdc.gov/mmwr/preview/ mmwrhtml/su6203a17.htm?s_cid= su6203a17_w\#Tab1. Accessed June 18, 2015

35. Centers for Disease Control and Prevention. National Center for Chronic Disease Prevention and Health Promotion. Overweight and 0besity 2014. Available at: http://www.cdc.gov/ obesity/index.html. Accessed June 18, 2015

36. Moher D, Liberati A, Tetzlaff J, Altman DG; PRISMA Group. Preferred reporting items for systematic reviews and meta-analyses: the PRISMA statement. PLoS Med. 2009;6(7):e1000097

37. Goy E, Kansagara D, Freeman M. USPSTF quality rating criteria for randomized controlled trials (RCTs) and cohort studies criteria. Available at: www.ncbi.n/m.nih.gov/books/ PMH0009125/. Accessed January 19, 2015

38. Bonuck K, Stuebe A, Barnett J, Labbok $\mathrm{MH}$, Fletcher J, Bernstein PS. Effect of primary care intervention on breastfeeding duration and intensity. Am J Public Health. 2014;104(Suppl 1):S119-S127

39. Howell EA, Bodnar-Deren S, Balbierz A, Parides M, Bickell N. An intervention to extend breastfeeding among black and Latina mothers after delivery. Am J Obstet Gynecol. 2014;210(3):239 e1-239.e5

40. Bonuck KA, Trombley M, Freeman K, McKee D. Randomized, controlled trial of a prenatal and postnatal lactation consultant intervention on duration and intensity of breastfeeding up to 12 months. Pediatrics. 2005;116(6):1413-1426

41. Chapman DJ, Morel K, Bermúdez-Millán A, Young S, Damio G, Pérez-Escamilla
R. Breastfeeding education and support trial for overweight and obese women: a randomized trial. Pediatrics. 2013;131(1):e162-e170

42. Petrova A, Ayers C, Stechna S, Gerling JA, Mehta R. Effectiveness of exclusive breastfeeding promotion in low-income mothers: a randomized controlled study. Breastfeed Med. 2009;4(2):63-69

43. Chapman DJ, Damio G, Young S, Pérez-Escamilla R. Effectiveness of breastfeeding peer counseling in a low-income, predominantly Latina population: a randomized controlled trial. Arch Pediatr Adolesc Med. 2004;158(9):897-902

44. Grassley JS, Sauls DJ. Evaluation of the supportive needs of adolescents during childbirth intrapartum nursing intervention on adolescents' childbirth satisfaction and breastfeeding rates. $\checkmark$ Obstet Gynecol Neonatal Nurs. 2012;41(1):33-44

45. Sandy JM, Anisfeld E, Ramirez E. Effects of a prenatal intervention on breastfeeding initiation rates in a Latina immigrant sample. J Hum Lact. 2009;25(4):404-411, quiz 458-459

46. Gill SL, Reifsnider E, Lucke JF. Effects of support on the initiation and duration of breastfeeding. West J Nurs Res. 2007;29(6):708-723

47. Anderson AK, Damio G, Young S, Chapman DJ, Pérez-Escamilla R. A randomized trial assessing the efficacy of peer counseling on exclusive breastfeeding in a predominantly Latina low-income community. Arch Pediatr Adolesc Med. 2005;159(9):836-841

48. Anderson AK, Damio G, Chapman DJ, Pérez-Escamilla R. Differential response to an exclusive breastfeeding peer counseling intervention: the role of ethnicity. J Hum Lact. 2007;23(1):16-23

49. Egger M, Smith GD, Altman D, eds. Systematic Reviews in Health Care: Meta-Analysis in Context. New York: John Wiley \& Sons; 2008

50. Mosteller F, Colditz GA. Understanding research synthesis (metaanalysis). Annu Rev Public Health. 1996;17(1):1-23 
51. Ludema C, Cole SR, Poole C, Chu

$\mathrm{H}$, Eron JJ. Meta-analysis of randomized trials on the association of prophylactic acyclovir and HIV-1 viral load in individuals coinfected with herpes simplex virus-2. AIDS. 2011;25(10):1265-1269

52. Higgins JPT, Thompson SG, Spiegelhalter DJ. A re-evaluation of random-effects meta-analysis. $J$ R Stat Soc Ser A Stat Soc. 2009;172(1):137-159

53. Borenstein M, Hedges L, Higgins J, Rothstein H. Introduction to MetaAnalysis. New York: John Wiley \& Sons; 2009

54. Riley RD, Higgins JP, Deeks JJ. Interpretation of random effects metaanalyses. BMJ. 2011;342:d549

55. Egger M, Davey Smith G, Schneider $\mathrm{M}$, Minder C. Bias in meta-analysis detected by a simple, graphical test. BMJ. 1997;315(7109):629-634
56. Begg CB, Mazumdar M. Operating characteristics of a rank correlation test for publication bias. Biometrics. 1994;50(4):1088-1101

57. Duval S, Tweedie R. Trim and fill: A simple funnel-plot-based method of testing and adjusting for publication bias in meta-analysis. Biometrics. 2000;56(2):455-463

58. Sussner KM, Lindsay AC, Peterson $K E$. The influence of acculturation on breast-feeding initiation and duration in low-income women in the US. J Biosoc Sci. 2008;40(5):673-696

59. Renfrew M, McCormick F, Wade A, Quinn B, Dowswell T. Support for healthy breastfeeding mothers with healthy term babies. Cochrane Database Syst Rev. 2012;16(5):CD001141

60. Ibanez G, de Reynal de Saint Michel C, Denantes M, SaurelCubizolles M-J, Ringa V, Magnier A-M. Systematic review and meta-analysis of randomized controlled trials evaluating primary care-based interventions to promote breastfeeding in low-income women. Fam Pract. 2012;29(3):245-254

61. Mann AR, Reifsnider E, Gill SL, Ritsema M. Health disparities in breastfeeding among low-income and Hispanic women. J Multicult Nurs Health. 2003;9(3):49-56

62. US Department of Health and Human Services. The Surgeon General's call to action to support breastfeeding. 2011. Available at: www.surgeongeneral.gov/ library/calls/breastfeeding/. Accessed June 18, 2015

63. US Preventive Services Task Force. Final Research Plan for Breastfeeding: Counseling, 2016. Available at: www. uspreventiveservicestaskforce.org/ Page/Document/ResearchPlanFinal /breastfeeding-primary-careinterventions. Accessed January 22, 2015 